PROCEEDINGS OF THE

AMERICAN MATHEMATICAL SOCIETY

Volume 127, Number 1, January 1999, Pages 35-44

S 0002-9939(99)04646-8

\title{
AN EXTENSION OF A CONVEXITY THEOREM OF THE GENERALIZED NUMERICAL RANGE ASSOCIATED WITH $S O(2 n+1)$
}

\author{
TIN-YAU TAM
}

(Communicated by Palle E. T. Jorgensen)

\begin{abstract}
For any $C, A_{1}, A_{2}, A_{3} \in \mathfrak{s o}(2 n+1)$, let $W_{C}\left(A_{1}, A_{2}, A_{3}\right)$ be the following subset of $\mathbb{R}^{3}$ :

$$
\left\{\left(\operatorname{tr} C O^{T} A_{1} O, \operatorname{tr} C O^{T} A_{2} O, \operatorname{tr} C O^{T} A_{3} O\right): O \in S O(2 n+1)\right\} .
$$

We show that if $n \geq 2$, then $W_{C}\left(A_{1}, A_{2}, A_{3}\right)$ is always convex. When $n=1$, it is an ellipsoid, probably degenerate. The convexity result is best possible in the sense that if we have $W_{C}\left(A_{1}, \ldots, A_{p}\right)$ defined similarly, then there are examples which fail to be convex when $p \geq 4$ and $n \geq 1$.

The set is also symmetric about the origin for all $n \geq 1$, and contains the origin when $n \geq 2$. Equivalent statements of this result are given. The convexity result for $\mathfrak{s o}(2 n+1)$ is similar to Au-Yeung and Tsing's extension of Westwick's convexity result for $\mathfrak{u}(n)$.
\end{abstract}

\section{INTRODUCTION}

Very recently the notion of numerical range has been generalized in the context of compact connected Lie groups [13]. See [13] and [6] for historical remarks. Let $G$ be a compact connected Lie group with Lie algebra $\mathfrak{g}$ which is equipped with a $G$-invariant inner product $\langle\cdot, \cdot\rangle$. For $A_{1}, \ldots, A_{p}, C \in \mathfrak{g}$, the $C$-numerical range of $\left(A_{1}, \ldots, A_{p}\right)$ is defined to be the following subset of $\mathbb{R}^{p}$ :

$$
W_{C}\left(A_{1}, \ldots, A_{p}\right)=\left\{\left(\left\langle A_{1}, Z\right\rangle, \ldots,\left\langle A_{p}, Z\right\rangle\right): Z \in O(C)\right\},
$$

where $O(C)=\{\operatorname{Ad}(g) C: g \in G\}$, the adjoint orbit of $C$.

Theorem 1 ([13]). Let $G$ be a compact connected Lie group. For $A_{1}, A_{2}, C \in \mathfrak{g}$, $W_{C}\left(A_{1}, A_{2}\right)$ is a compact convex set in $\mathbb{R}^{2}$.

Corollary 2. 1. [15] If $G=U(n)$ or $S U(n)$, then

$$
W_{C}\left(A_{1}, A_{2}\right)=\left\{\left(\operatorname{tr} A_{1} U^{*} C U, \operatorname{tr} A_{2} U^{*} C U\right): U \in G\right\}
$$

is convex, where $A_{1}, A_{2}$ and $C$ are Hermitian matrices.

2. The set $W_{C}\left(A_{1}, A_{2}\right)=\left\{\left(\operatorname{tr} A_{1} O^{T} C O, \operatorname{tr} A_{2} O^{T} C O\right): O \in S O(n)\right\}$ is convex, where $A_{1}, A_{2}$, and $C$ are real skew symmetric matrices.

Received by the editors November 26, 1996 and, in revised form, May 9, 1997.

1991 Mathematics Subject Classification. Primary 15A60, 22E15.

Key words and phrases. Numerical range, convexity, special orthogonal group, weak majorization. 
Theorem 1 is best possible in the sense that $W_{C}\left(A_{1}, \ldots, A_{p}\right)$ fails to be convex when $p>2$, e.g., convexity fails to hold if $p>3$ or $n=2$ when $G=U(n)$ [2], and if $p=3$ and $n=1$ when $G=S O(2 n+1)$ [14]. Nevertheless, there are equivalent statements for the convexity of $W_{C}\left(A_{1}, \ldots, A_{p}\right)$ [13]. In order to state the result, we need to introduce some notations. Let $\mathfrak{t}$ be the Lie algebra of a maximal torus $T$ of the compact connected Lie group $G$. We denote by $W$ the Weyl group of $G$ and by $\hat{K}$ the convex hull of the set $K$. Since every adjoint orbit intersects $\mathfrak{t}$ in a finite non-empty set, we can assume [13] that $C \in \mathfrak{t}$ for $W_{C}\left(A_{1}, \ldots, A_{p}\right)$ or even $C \in \mathcal{C}$, where $\mathcal{C}$ is the (closed) fundamental Weyl chamber.

Theorem 3 ([13]). Let $A_{1}, \ldots, A_{p}$ be elements in $\mathfrak{g}$ and let $C \in \mathfrak{t}$. The following statements are equivalent:

1. $W_{C}\left(A_{1}, \ldots, A_{p}\right)$ is convex.

2. If $\left(r_{1}, \ldots, r_{p}\right) \in \mathbb{R}^{p} \backslash W_{C}\left(A_{1}, \ldots, A_{p}\right)$, then there exist $\alpha_{1}, \ldots, \alpha_{p} \in \mathbb{R}$ such that $\min _{g \in G}\left\langle\sum_{i=1}^{p} \alpha_{i} A_{i}, \operatorname{Ad}(g) C\right\rangle>\sum_{i=1}^{p} \alpha_{i} r_{i}$.

3. If $\left(r_{1}, \ldots, r_{p}\right) \in \mathbb{R}^{p} \backslash W_{C}\left(A_{1}, \ldots, A_{p}\right)$, then there exist $\alpha_{1}, \ldots, \alpha_{p} \in \mathbb{R}$ such that $\min _{\omega \in W}\langle\omega \cdot \tilde{A}, C\rangle>\sum_{i=1}^{p} \alpha_{i} r_{i}$, where $\tilde{A} \in O(A) \cap \mathcal{C}$ and $A=\sum_{i=1}^{p} \alpha_{i} A_{i}$.

4. $W_{B}\left(A_{1}, \ldots, A_{p}\right) \subset W_{C}\left(A_{1}, \ldots, A_{p}\right)$ if $B \in \mathfrak{t}$ and $B \in \widehat{W(C)}$.

While Westwick's convexity result [15] implies that the statements of Theorem 3 $(G=U(n))$ are valid if $p=2, \mathrm{Au}$-Yeung and Tsing [4] proved that the statements of Theorem 3 are true when $p=3$ and $n>2(G=U(n))$. This obviously extends Westwick's result when $n>2$. See [11] for further generalization. When $n=2$, it can be shown that $W_{C}\left(A_{1}, A_{2}, A_{3}\right)$ is an ellipsoid and hence is not convex in general.

In this paper, we deal with another special case, namely, $S O(2 n+1)$ when $p=3$ and $n \geq 2$.

When $G=S O(2 n+1)$, let $\mathfrak{t}$ be the space of skew symmetric matrices of the form

$$
\left(\begin{array}{cc}
0 & c_{1} \\
-c_{1} & 0
\end{array}\right) \oplus \cdots \oplus\left(\begin{array}{cc}
0 & c_{n} \\
-c_{n} & 0
\end{array}\right) \oplus 0
$$

Every $C \in \mathfrak{s o}(2 n+1)$ has the canonical form (1), i.e., there exist $O \in S O(2 n+1)$ such that $O^{T} C O$ is in $\mathfrak{t}$. We can identify $\mathfrak{t}$ with $\mathbb{R}^{n}$ by sending $(1)$ to $\left(c_{1}, \ldots, c_{n}\right)$. So the (closed) fundamental Weyl chamber $\mathcal{C}$ can be identified with $\mathbb{R}_{+}^{n}=\left\{\left(x_{1}, \ldots, x_{n}\right)\right.$ $\left.\in \mathbb{R}^{n}, x_{1} \geq x_{2} \geq \cdots \geq x_{n} \geq 0\right\}$. The Weyl group $W$ operates on $\mathfrak{t}$, and its action is given by $\left(c_{1}, \ldots, c_{n}\right) \mapsto\left( \pm c_{\theta(1)}, \ldots, \pm c_{\theta(n)}\right)$, where $\theta \in \Sigma_{n}$, and for any choice of sign.

Let $C$ and $B$ be skew symmetric matrices with canonical forms corresponding to $c$ and $b$ respectively, according to (1). Then $B \in \widehat{W(C)}$ amounts to the condition [12] that $b$ is in the convex hull of the vectors $\left( \pm c_{\theta(1)}, \ldots, \pm c_{\theta(n)}\right)$, where $\theta \in \Sigma_{n}$, and for any choice of sign. This is equivalent to saying that $\sum_{i=1}^{k}\left|b_{i}\right| \leq \sum_{i=1}^{k}\left|c_{i}\right|$, $k=1, \ldots, n$, after rearranging the entries of $b$ and $c$ in descending order of absolute values. We will denote the relation by $y \prec_{w} x$. If $b$ and $c$ are both nonnegative vectors, then the inequalities become the usual weak majorization [7] for $\mathbb{R}_{+}^{n}$.

With respect to $W_{C}\left(A_{1}, \ldots, A_{p}\right), C$ can be assumed in the form (1), where $c \in \mathbb{R}_{+}^{n}$. We can even assume that $C \in \mathcal{C}$. The reason is that

$$
W_{C}\left(A_{1}, \ldots, A_{p}\right)=W_{P^{T} C P}\left(A_{1}, \ldots, A_{p}\right) \quad \text { for any } P \in S O(2 n+1) .
$$


Corollary 4 ([13]). Let $C, A_{1}, \ldots, A_{p} \in \mathfrak{s o}(2 n+1)$ and let $C$ be in the canonical form (1) corresponding to $c=\left(c_{1}, \ldots, c_{n}\right) \in \mathbb{R}_{+}^{n}$. The following statements are equivalent:

1. $W_{C}\left(A_{1}, \ldots, A_{p}\right)$ is convex.

2. If $\left(r_{1}, \ldots, r_{p}\right) \in \mathbb{R}^{p} \backslash W_{C}\left(A_{1}, \ldots, A_{p}\right)$, then there exist $\alpha_{1}, \ldots, \alpha_{p} \in \mathbb{R}$ such that

$$
\min _{O \in S O(2 n+1)} \operatorname{tr} C O^{T}\left(\sum_{i=1}^{p} \alpha_{i} A_{i}\right) O>\sum_{i=1}^{p} \alpha_{i} r_{i} .
$$

3. If $\left(r_{1}, \ldots, r_{p}\right) \in \mathbb{R}^{p} \backslash W_{C}\left(A_{1}, \ldots, A_{p}\right)$, then there exist $\alpha_{1}, \ldots, \alpha_{p} \in \mathbb{R}$ such that

$$
\min _{\sigma \in \Sigma_{n}}-2 \sum_{i=1}^{n} c_{i} a_{\sigma(i)}>\sum_{i=1}^{p} \alpha_{i} r_{i}
$$

where the canonical form of $\sum_{i=1}^{p} \alpha_{i} A_{i}$ corresponds to $a=\left(a_{1}, \ldots, a_{n}\right) \in \mathbb{R}_{+}^{n}$, according to (1).

4. $W_{B}\left(A_{1}, \ldots, A_{p}\right) \subset W_{C}\left(A_{1}, \ldots, A_{p}\right)$ if $b \prec_{w} c$, where $b \in \mathbb{R}_{+}^{n}$ and the canonical form of $B$ corresponds to $b=\left(b_{1}, \ldots, b_{n}\right)$, according to (1).

\section{Convexity And SOME EQUivalent STATEMEnts}

Mirsky [9] obtained the following result, which was redicovered by Chong [5]. Also see [7].

Lemma 5. Let $x, y \in \mathbb{R}^{n}$. Then $y \prec_{w} x$ if and only if $y \leq P_{1} \cdots P_{k} x$ for some pinching matrices $P_{1}, \ldots, P_{k}$. Hence, if $x, y \in \mathbb{R}_{+}^{n}$, then $y \prec_{w} x$ if and only if $y=\Gamma P_{1} \cdots P_{k} x$ for some pinching matrices $P_{1}, \ldots, P_{k}$ and $\Gamma=\operatorname{diag}\left(\gamma_{1}, \ldots, \gamma_{n}\right)$ with $0 \leq \gamma_{i} \leq 1, i=1, \ldots, n$.

Lemma 6. Let $C \neq 0$ and $A_{1}, A_{2}, A_{3} \in \mathfrak{s o}(3)$, where

$$
A_{1}=\left(\begin{array}{ccc}
0 & x_{1} & x_{2} \\
-x_{1} & 0 & x_{3} \\
-x_{2} & -x_{3} & 0
\end{array}\right), \quad A_{2}=\left(\begin{array}{ccc}
0 & y_{1} & y_{2} \\
-y_{1} & 0 & y_{3} \\
-y_{2} & -y_{3} & 0
\end{array}\right), \quad A_{3}=\left(\begin{array}{ccc}
0 & z_{1} & z_{2} \\
-z_{1} & 0 & z_{3} \\
-z_{2} & -z_{3} & 0
\end{array}\right)
$$

$$
A=\left(\begin{array}{lll}
x_{1} & x_{2} & x_{3} \\
y_{1} & y_{2} & y_{3} \\
z_{1} & z_{2} & z_{3}
\end{array}\right)
$$

Then $W_{C}\left(A_{1}, A_{2}, A_{3}\right)=\left\{\left(\operatorname{tr} C O^{T} A_{1} O, \operatorname{tr} C O^{T} A_{2} O, \operatorname{tr} C O^{T} A_{3} O\right): O \in S O(3)\right\} \subset$ $\mathbb{R}^{3}$ is

1. a nondegenerate ellipsoid centered at the origin, if $\operatorname{rank} A=3$;

2. an elliptical disk centered at the origin, if $\operatorname{rank} A=2$;

3. a line segment centered at the origin, if $\operatorname{rank} A=1$;

4. the origin if $\operatorname{rank} A=0$.

Proof. By using the technique in [14], it can be deduced that the ellipsoid $\mathcal{E}$ in Lemma 6 is given in the following way: Let $A=O_{1} \Lambda O_{2}$ be a singular value decomposition of $A$, where $O_{1} \in S O(3)$. Let $r S^{2}$ be the 2 -sphere centered at the origin and with radius $r=\sqrt{\frac{1}{2} C^{T} C}$. The ellipsoid $\mathcal{E}$ is $O_{1}\left(\mathcal{E}^{\prime}\right)$ where $\mathcal{E}^{\prime}=$ $\Lambda O_{2}\left(r S^{2}\right)=\Lambda\left(r S^{2}\right)$ is the ellipsoid in the standard position, centered at the origin and principal axes $x_{1}, x_{2}$ and $x_{3}$, respectively. The principal axes lengths are $\lambda_{1}$, $\lambda_{2}$ and $\lambda_{3}$, respectively, where $\Lambda=\operatorname{diag}\left(\lambda_{1}, \lambda_{2}, \lambda_{3}\right)$. 
The following is the main result of this paper.

Theorem 7. Let $C, A_{1}, A_{2}, A_{3} \in \mathfrak{s o}(2 n+1)$ and let $C$ be in the canonical form (1) corresponding to $c=\left(c_{1}, \ldots, c_{n}\right) \in \mathbb{R}_{+}^{n}$. The following statements hold.

1. $W_{C}\left(A_{1}, A_{2}, A_{3}\right)$ is convex.

2. If $\left(r_{1}, r_{2}, r_{3}\right) \in \mathbb{R}^{3} \backslash W_{C}\left(A_{1}, A_{2}, A_{3}\right)$, then there exist $\alpha_{1}, \alpha_{2}, \alpha_{3} \in \mathbb{R}$ such that

$$
\min _{O \in S O(2 n+1)} \operatorname{tr} C O^{T}\left(\sum_{i=1}^{n} \alpha_{i} A_{i}\right) O>\alpha_{1} r_{1}+\alpha_{2} r_{2}+\alpha_{3} r_{3} .
$$

3. If $\left(r_{1}, r_{2}, r_{3}\right) \in \mathbb{R}^{3} \backslash W_{C}\left(A_{1}, A_{2}, A_{3}\right)$, then there exist $\alpha_{1}, \alpha_{2}, \alpha_{3} \in \mathbb{R}$ such that

$$
\min _{\sigma \in \Sigma_{n}}-2 \sum_{i=1}^{n} c_{i} a_{\sigma(i)}>\alpha_{1} r_{1}+\alpha_{2} r_{2}+\alpha_{3} r_{3}
$$

where the canonical form of $\alpha_{1} A_{1}+\alpha_{2} A_{2}+\alpha_{3} A_{3}$ corresponds to $a=\left(a_{1}, \ldots, a_{n}\right)$ $\in \mathbb{R}_{+}^{n}$, according to (1).

4. $W_{B}\left(A_{1}, A_{2}, A_{3}\right) \subset W_{C}\left(A_{1}, A_{2}, A_{3}\right)$ if $b \prec_{w} c$, and $B \in \mathfrak{s o}(2 n+1)$ has canonical form corresponding to $b=\left(b_{1}, \ldots, b_{n}\right) \in \mathbb{R}_{+}^{n}$ according to (1).

Proof. We are going to use the continuity argument [4] to furnish the proof. Due to Corollary 4, it suffices to show that the last statement of Theorem 7 is valid. Because of Lemma 5 , it is sufficent to establish that $W_{B}\left(A_{1}, A_{2}, A_{3}\right) \subset W_{C}\left(A_{1}, A_{2}, A_{3}\right)$ when

1. $b=\Gamma c$, where $\Gamma=\operatorname{diag}(1, \ldots, 1, \alpha, 1, \ldots, 1), 0 \leq \alpha<1$; and

2. $b=P c$, where $P$ is a pinching matrix.

Due to (2), we only have to deal with the following two cases:

Case 1. $b=\Gamma c$, where $b, c \in \mathbb{R}_{+}^{n}$, and

$$
\Gamma=\operatorname{diag}(\alpha, 1, \ldots, 1), \quad 0 \leq \alpha<1 .
$$

Hence we can assume that $C$ and $B$ are of the form (1) corresponding to $c$ and $b$ respectively, where $0 \leq b_{1}<c_{1}$ and $c_{i}=b_{i}$ for $i=2, \ldots, n$. Suppose that $x=\left(x_{1}, x_{2}, x_{3}\right) \in W_{B}\left(A_{1}, A_{2}, A_{3}\right)$, i.e., there exists $E \in S O(2 n+1)$ such that

$$
x=\left(\operatorname{tr} B E^{T} A_{1} E, \operatorname{tr} B E^{T} A_{2} E, \operatorname{tr} B E^{T} A_{3} E\right) .
$$

Let $e_{1}, e_{2}, \ldots, e_{2 n+1}$ be the columns of $E$. Then

$$
x_{i}=-2\left(b_{1} e_{1}^{T} A_{i} e_{2}+\sum_{j=2}^{n} b_{j} e_{2 j-1}^{T} A_{i} e_{2 j}\right), \quad i=1,2,3 .
$$

The point $\gamma=-2 b_{1}\left(e_{1}^{T} A_{1} e_{2}, e_{1}^{T} A_{2} e_{2}, e_{1}^{T} A_{3} e_{2}\right)$ belongs to $W_{B^{\prime}}\left(A_{1}^{\prime}, A_{2}^{\prime}, A_{3}^{\prime}\right)$, which is an ellipsoid (probably degenerate) centered at the origin, by Lemma 6 , where $A_{i}^{\prime}=\left(E^{T} A_{i} E\right)[1,2,2 n+1 \mid 1,2,2 n+1], i=1,2,3$, are elements of $\mathfrak{s o}(3)$ and $A[\alpha \mid \beta]$ denotes the submatrix of $A$ lying in the rows given by the sequence $\alpha$ and in the columns given by the sequence $\beta$ and

$$
B^{\prime}=\left(\begin{array}{cc}
0 & b_{1} \\
-b_{1} & 0
\end{array}\right) \oplus 0
$$

If we denote by $\mathcal{E}_{b_{1}, E}$ the ellipsoid obtained by translating $W_{B^{\prime}}\left(A_{1}^{\prime}, A_{2}^{\prime}, A_{3}^{\prime}\right)$ by

$$
-2\left(\sum_{j=2}^{n} b_{j} e_{2 j-1}^{T} A_{1} e_{2 j}, \sum_{j=2}^{n} b_{j} e_{2 j-1}^{T} A_{2} e_{2 j}, \sum_{j=2}^{n} b_{j} e_{2 j-1}^{T} A_{3} e_{2 j}\right),
$$

then $x \in \mathcal{E}_{b_{1}, E} \subset W_{B}\left(A_{1}, A_{2}, A_{3}\right)$. 
Similarly we have the ellipsoid $\mathcal{E}_{c_{1}, E} \subset W_{C}\left(A_{1}, A_{2}, A_{3}\right)$. Since $0 \leq b_{1}<c_{1}$, $b_{j}=c_{j}, j=2, \ldots, n$, the ellipsoid $W_{B^{\prime}}\left(A_{1}^{\prime}, A_{2}^{\prime}, A_{3}^{\prime}\right)$ lies within the interior of the ellipsoid $W_{C^{\prime}}\left(A_{1}^{\prime}, A_{2}^{\prime}, A_{3}^{\prime}\right)$, where

$$
C^{\prime}=\left(\begin{array}{cc}
0 & c_{1} \\
-c_{1} & 0
\end{array}\right) \oplus 0
$$

Notice that $\mathcal{E}_{c_{1}, E}$ is the ellipsoid obtained by translating $W_{C^{\prime}}\left(A_{1}^{\prime}, A_{2}^{\prime}, A_{3}^{\prime}\right)$ by

$$
-2\left(\sum_{j=2}^{n} c_{j} e_{2 j-1}^{T} A_{1} e_{2 j}, \sum_{j=2}^{n} c_{j} e_{2 j-1}^{T} A_{2} e_{2 j}, \sum_{j=2}^{n} c_{j} e_{2 j-1}^{T} A_{3} e_{2 j}\right),
$$

which is identical to

$$
-2\left(\sum_{j=2}^{n} b_{j} e_{2 j-1}^{T} A_{1} e_{2 j}, \sum_{j=2}^{n} b_{j} e_{2 j-1}^{T} A_{2} e_{2 j}, \sum_{j=2}^{n} b_{j} e_{2 j-1}^{T} A_{3} e_{2 j}\right) .
$$

So $\mathcal{E}_{b_{1}, E}$ lies within the interior of $\mathcal{E}_{c_{1}, E}$. If $W_{C^{\prime}}\left(A_{1}^{\prime}, A_{2}^{\prime}, A_{3}^{\prime}\right)$ degenerates, so do $\mathcal{E}_{c_{1}, E}$ and $\mathcal{E}_{b_{1}, E}$. In this case, $x \in \mathcal{E}_{b_{1}, E} \subset \mathcal{E}_{c_{1}, E} \subset W_{C}\left(A_{1}, A_{2}, A_{3}\right)$. Thus we assume that $W_{C^{\prime}}\left(A_{1}^{\prime}, A_{2}^{\prime}, A_{3}^{\prime}\right)$ does not degenerate.

Now pick two orthonormal vectors $z_{1}$ and $z_{2}$ in $\mathbb{R}^{2 n+1}$ such that $\operatorname{span}\left\{z_{1}, z_{2}\right\}$ is an invariant subspace of $A_{1}$. Since $n \geq 2$, there exists $z_{2 n+1} \in \mathbb{R}^{2 n+1}$ such that $z_{2 n+1}$ is perpendicular to the four vectors $z_{1}, z_{2}, A_{2} z_{1}$ and $A_{2} z_{2}$. Then extend $\left\{z_{1}, z_{2}, z_{2 n+1}\right\}$ to an orthonormal basis $\left\{z_{1}, z_{2}, z_{3}, \ldots, z_{2 n}, z_{2 n+1}\right\}$ of $\mathbb{R}^{2 n+1}$ so that the matrix $Z=\left(\begin{array}{llll}z_{1} & z_{2} & \ldots & z_{2 n+1}\end{array}\right)$ is an element of $S O(2 n+1)$. Then the two matrices $\left(Z^{T} A_{1} Z\right)[1,2,2 n+1 \mid 1,2,2 n+1],\left(Z^{T} A_{2} Z\right)[1,2,2 n+1 \mid 1,2,2 n+1]$ are of the following form:

$$
\left(\begin{array}{lll}
0 & * & 0 \\
* & 0 & 0 \\
0 & 0 & 0
\end{array}\right)
$$

According to Lemma 6 , the ellipsoid $\mathcal{E}_{c_{1}, Z}$ degenerates. Let $Z^{\prime}, E^{\prime} \in \mathfrak{s o}(2 n+1)$ such that $Z=e^{Z^{\prime}}$ and $E=e^{E^{\prime}}$. Then consider a continuous path $F(t)$ in $S O(2 n+1)$ defined by $F(t)=e^{t Z^{\prime}+(1-t) E^{\prime}}$, where $0 \leq t \leq 1$. Notice that $\mathcal{E}_{c_{1}, F(t)} \subset W_{C}\left(A_{1}, A_{2}, A_{3}\right)$ for all $t \in[0,1]$. Now $x$ lies within the interior of $\mathcal{E}_{c_{1}, F(0)} \equiv \mathcal{E}_{c_{1}, E}$. Moreover, $\mathcal{E}_{c_{1}, F(1)} \equiv \mathcal{E}_{c_{1}, Z}$ degenerates and is then convex. So by the continuity argument, as in [4] there exists $t \in[0,1]$ such that $x \in \mathcal{E}_{c_{1}, F(t)}$. Thus $x \in W_{C}\left(A_{1}, A_{2}, A_{3}\right)$.

Case 2. $b=P c$, where $b, c \in \mathbb{R}_{+}^{n}$, and

$$
P=\alpha I+(1-\alpha) Q, \quad 0<\alpha<1, \quad \text { and } \quad Q=\left(\begin{array}{cc}
0 & 1 \\
1 & 0
\end{array}\right) \oplus I_{n-2} .
$$

We can also assume that $c_{1}>c_{2}$ and $b_{1} \geq b_{2}$. In other words, we have $b=$ $\left(b_{1}, b_{2}, \ldots, b_{n}\right), c=\left(c_{1}, c_{2}, \ldots, c_{n}\right) \in \mathbb{R}_{+}^{n}$ such that

$$
b_{1}+b_{2}=c_{1}+c_{2}, \quad 0 \leq b_{1}-b_{2}<c_{1}-c_{2}, \quad b_{i}=c_{i}, \quad i=3, \ldots, n .
$$

Because of (2), we can assume that $C$ and $B$ are in the form (1) corresponding to $c$ and $b$ respectively. Suppose that $x=\left(x_{1}, x_{2}, x_{3}\right) \in W_{B}\left(A_{1}, A_{2}, A_{3}\right)$, i.e., there exists $E \in S O(2 n+1)$ such that

$$
x=\left(\operatorname{tr} B E^{T} A_{1} E, \operatorname{tr} B E^{T} A_{2} E, \operatorname{tr} B E^{T} A_{3} E\right) .
$$


Let $e_{1}, e_{2}, \ldots, e_{2 n+1}$ be the columns of $E$. Then for $i=1,2,3$,

$$
\begin{aligned}
x_{i}= & -2 b_{1} e_{1}^{T} A_{i} e_{2}-2 b_{2} e_{3}^{T} A_{i} e_{4}-2 \sum_{j=3}^{n} b_{j} e_{2 j-1}^{T} A_{i} e_{2 j} \\
= & -\left(b_{1}+b_{2}\right)\left(e_{1}^{T} A_{i} e_{2}+e_{3}^{T} A_{i} e_{4}\right) \\
& -\left(b_{1}-b_{2}\right)\left(e_{1}^{T} A_{i} e_{2}-e_{3}^{T} A_{i} e_{4}\right)-2 \sum_{j=3}^{n} b_{j} e_{2 j-1}^{T} A_{i} e_{2 j} .
\end{aligned}
$$

Let $f_{1}, f_{2}, f_{3}$ and $f_{4} \in \mathbb{R}^{2 n+1}$ be the vectors defined by the relation:

$$
\begin{aligned}
& f_{1}+i f_{2}=e^{-i \phi} \cos \theta\left(e_{1}+i e_{2}\right)+e^{i \phi} \sin \theta\left(e_{3}+i e_{4}\right), \\
& f_{3}+i f_{4}=-e^{-i \phi} \sin \theta\left(e_{1}+i e_{2}\right)+e^{i \phi} \cos \theta\left(e_{3}+i e_{4}\right) .
\end{aligned}
$$

The matrix which sends $\left(e_{1}, e_{2}, e_{3}, e_{4}\right)$ to $\left(f_{1}, f_{2}, f_{3}, f_{4}\right)$ is an element of $S O(4)$ :

$$
\left(\begin{array}{cccc}
\cos \phi \cos \theta & -\sin \phi \cos \theta & -\cos \phi \sin \theta & \sin \phi \sin \theta \\
\sin \phi \cos \theta & \cos \phi \cos \theta & -\sin \phi \sin \theta & -\cos \phi \sin \theta \\
\cos \phi \sin \theta & \sin \phi \sin \theta & \cos \phi \cos \theta & \sin \phi \cos \theta \\
-\sin \phi \sin \theta & \cos \phi \sin \theta & -\sin \phi \cos \theta & \cos \phi \cos \theta
\end{array}\right) \text {. }
$$

So $f_{1}, f_{2}, f_{3}, f_{4} \in \mathbb{R}^{2 n+1}$ are orthonormal vectors and the matrix

$$
F=\left(\begin{array}{lllllll}
f_{1} & f_{2} & f_{3} & f_{4} & e_{5} & \cdots & e_{2 n+1}
\end{array}\right)
$$

is an element of $S O(2 n+1)$. It is clear that $\left(e_{1}+i e_{2}\right)^{*} A_{j}\left(e_{1}+i e_{2}\right)=2 i e_{1}^{T} A_{j} e_{2}$ since $A_{j}$ is real skew symmetric. By direct computation, we have

$$
f_{1}^{T} A_{j} f_{2}+f_{3}^{T} A_{j} f_{4}=e_{1}^{T} A_{j} e_{2}+e_{3}^{T} A_{j} e_{4}, \quad j=1,2,3,
$$

and

$$
f_{1}^{T} A_{j} f_{2}-f_{3}^{T} A_{j} f_{4}=p_{j} \cos 2 \theta+\sin 2 \theta\left(q_{j} \sin 2 \phi+s_{j} \cos 2 \phi\right), \quad j=1,2,3,
$$

where

$$
p_{j}=e_{1}^{T} A_{j} e_{2}-e_{3}^{T} A_{j} e_{4}, \quad q_{j}=e_{1}^{T} A_{j} e_{3}-e_{2}^{T} A_{j} e_{4}, \quad s_{j}=-e_{2}^{T} A_{j} e_{3}+e_{1}^{T} A_{j} e_{4} .
$$

If we set $y_{i}=\operatorname{tr} B F^{T} A_{i} F$, then for $i=1,2,3$,

$$
\begin{aligned}
y_{i}= & -\left(b_{1}+b_{2}\right)\left(f_{1}^{T} A_{i} f_{2}+f_{3}^{T} A_{i} e_{4}\right) \\
& -\left(b_{1}-b_{2}\right)\left(f_{1}^{T} A_{i} f_{2}-f_{3}^{T} A_{i} e_{4}\right)-2 \sum_{j=3}^{n} b_{j} e_{2 j-1}^{T} A_{i} e_{2 j} \\
= & -\left(b_{1}+b_{2}\right)\left(e_{1}^{T} A_{i} e_{2}+e_{3}^{T} A_{i} e_{4}\right) \\
& -\left(b_{1}-b_{2}\right)\left[p_{j} \cos 2 \theta+\sin 2 \theta\left(q_{j} \sin 2 \phi+s_{j} \cos 2 \phi\right)\right]-2 \sum_{j=3}^{n} b_{j} e_{2 j-1}^{T} A_{i} e_{2 j} .
\end{aligned}
$$

As $\theta$ and $\phi$ vary in $\mathbb{R}$, the locus of the point $\left(y_{1}, y_{2}, y_{3}\right)$ in $\mathbb{R}^{3}$ is an ellipsoid (compare [4]) which is denoted by $\mathcal{E}_{b_{1}, b_{2}, E}$. Indeed it is the translation of the ellipsoid (centered at the origin; $(\theta, \phi)$ and $(\pi+\theta, \phi)$ give opposite points on the ellipsoid) generated by the middle term. The ellipsoid $\mathcal{E}_{b_{1}, b_{2}, E}$ contains the point $x=\left(x_{1}, x_{2}, x_{3}\right)$, since $x$ corresponds to $\theta=0$ and $\phi=\pi / 2$. Similarly we have $\mathcal{E}_{c_{1}, c_{2}, E}$. Since $c_{1}+c_{2}=b_{1}+b_{2}$ and $0 \leq b_{1}-b_{2}<c_{1}-c_{2}$, we conclude that $\mathcal{E}_{b_{1}, b_{2}, E}$ lies within the 
interior of $\mathcal{E}_{c_{1}, c_{2}, E}$. Let $\pm i \lambda_{1}, \ldots, \pm i \lambda_{n}, 0$ be the eigenvalues of the skew symmetric matrix $A_{1}$, where $\lambda$ 's are nonnegative. Let

$$
Z=\left(\begin{array}{llll}
z_{1} & z_{2} & \ldots & z_{2 n+1}
\end{array}\right) \in S O(2 n+1)
$$

be such that

$$
Z^{T} A_{1} Z=\left(\begin{array}{cc}
0 & \lambda_{1} \\
-\lambda_{1} & 0
\end{array}\right) \oplus\left(\begin{array}{cc}
0 & \lambda_{2} \\
-\lambda_{2} & 0
\end{array}\right) \oplus \cdots \oplus\left(\begin{array}{cc}
0 & \lambda_{n} \\
-\lambda_{n} & 0
\end{array}\right) \oplus 0
$$

In particular, we have

$$
\begin{aligned}
& A_{1} z_{1}=\lambda_{1} z_{2}, \quad A_{1} z_{2}=-\lambda_{1} z_{1}, \\
& A_{1} z_{3}=\lambda_{2} z_{4}, \quad A_{1} z_{4}=-\lambda_{2} z_{3},
\end{aligned}
$$

and thus

$$
z_{2}^{T} A_{1} z_{3}=z_{1}^{T} A_{1} z_{4}=0, \quad z_{1}^{T} A_{1} z_{3}=z_{2}^{T} A_{1} z_{4} .
$$

If $\lambda_{1}=\lambda_{2}$, then $z_{1}^{T} A_{1} z_{2}-z_{3}^{T} A_{1} z_{4}=0$. The ellipsoid $\mathcal{E}_{c_{1}, c_{2}, Z}$ degenerates and lies within the $y z$ plane. By applying the continuity argument in case 1 to the matrices $E$ and $Z$, we have $x \in W_{C}\left(A_{1}, A_{2}, A_{3}\right)$. So we can assume that $\lambda_{1}, \ldots, \lambda_{n}$ are distinct and $\lambda_{1}>\lambda_{2}$. Let

$$
A_{1}^{\prime}=\left(Z^{T} A_{1} Z\right)[1,2,2 n+1 \mid 1,2,2 n+1]=\left(\begin{array}{ccc}
0 & \lambda_{1} & 0 \\
-\lambda_{1} & 0 & 0 \\
0 & 0 & 0
\end{array}\right)=P^{T} A_{1} P \in \mathfrak{s o}(3),
$$

where $P=\left(\begin{array}{lll}z_{1} & z_{2} & z_{2 n+1}\end{array}\right)$.

It is well known that the adjoint orbits, $\left\{O X O^{T}: O \in S O(3)\right\}$ for all $X \in \mathfrak{s o}(3)$, can be identified (using $\operatorname{tr} X^{T} Y$ as the inner product on $\mathfrak{s o}(3)$ ) with the 2-spheres in $\mathbb{R}^{3}$ centered at 0 and with radius $\sqrt{\frac{1}{2} \operatorname{tr} X^{T} X}$. So there exists $O \in S O(3)$ such that $\left(\begin{array}{lll}z_{1}^{\prime} & z_{2}^{\prime} & z_{2 n+1}^{\prime}\end{array}\right)=\left(\begin{array}{lll}z_{1} & z_{2} & z_{2 n+1}\end{array}\right) O$ and $z_{1}^{\prime T} A_{1}^{\prime} z_{2}^{\prime}=z_{3}^{T} A_{1}^{\prime} z_{4}=\lambda_{2}$, since $\lambda_{1}>\lambda_{2} \geq 0$. In other words,

$$
Z^{\prime T} A_{1}^{\prime} Z^{\prime}=\left(\begin{array}{ccccccc}
0 & \lambda_{2} & 0 & 0 & \ldots & 0 & u \\
-\lambda_{2} & 0 & 0 & 0 & \ldots & 0 & v \\
0 & 0 & 0 & \lambda_{2} & 0 & \ldots & 0 \\
0 & 0 & -\lambda_{2} & 0 & 0 & \ldots & 0 \\
& & \ldots & & & & \\
-u & -v & 0 & 0 & \ldots & 0 & 0
\end{array}\right), \quad u^{2}+v^{2}+\lambda_{2}^{2}=\lambda_{1}^{2},
$$

where $Z^{\prime}=\left(\begin{array}{lllllll}z_{1}^{\prime} & z_{2}^{\prime} & z_{3} & z_{4} & \ldots & z_{2 n} & z_{2 n+1}^{\prime}\end{array}\right)$. It follows that

$$
z_{2}^{\prime T} A_{1}^{\prime} z_{3}=z_{1}^{\prime T} A_{1} z_{4}=z_{1}^{\prime T} A_{1}^{\prime} z_{3}=z_{2}^{\prime T} A_{1} z_{4}=0, \quad z_{1}^{\prime T} A_{1}^{\prime} z_{2}^{\prime}=z_{3}^{T} A_{1} z_{4}
$$

So the corresponding $p_{1}, q_{1}$ and $s_{1}$ in (5) are all zeros. Thus the ellipsoid $\mathcal{E}_{c_{1}, c_{2}, Z^{\prime}}$ degenerates and lies within the $y z$ plane. Applying the continuity argument again, we are done.

Corollary 8. Let $C, A_{1}, A_{2}, A_{3} \in \mathfrak{s o}(2 n+1)$ and let $\alpha \in\left\{\operatorname{tr} C O^{T} A_{3} O: O \in\right.$ $S O(2 n+1)\} \subset \mathbb{R}$. Then in $\mathbb{R}^{2}$ the subset

$$
\left\{\left(\operatorname{tr} C O^{T} A_{1} O, \operatorname{tr} C O^{T} A_{2} O\right): O \in S O(2 n+1), \operatorname{tr} C O^{T} A_{3} O=\alpha\right\}
$$

is either empty or convex. 
Remark. We remark that the above results are valid if $G=O(2 n+1)(\mathfrak{o}(k) \equiv \mathfrak{s o}(k)$ for all $k$ ) whenever they are true for $G=S O(2 n+1)$, since $G=S O(2 n+1)$ and $O(2 n+1)$ give the same $W_{C}\left(A_{1}, \ldots, A_{p}\right)$.

Due to case 1 in the above proof, we conclude that $W_{C}\left(A_{1}, A_{2}, A_{3}\right)$ always contains the origin when $n \geq 2$, since $0 \prec_{w} c$ for $c \in \mathbb{R}_{+}^{n}$. One can deduce the same conclusion by the convexity of $W_{C}\left(A_{1}, A_{2}, A_{3}\right)$ when $n \geq 2$. The following symmetry result of $W_{C}\left(A_{1}, A_{2}, A_{3}\right)$ follows directly from the remark on the Weyl group.

Proposition 9. Let $G=S O(2 n+1)$ or $O(2 n+1)$ and $C, A_{1}, \ldots, A_{p} \in \mathfrak{s o}(2 n+1)$. Then $W_{C}\left(A_{1}, \ldots, A_{p}\right)$ is symmetric about the origin, i.e., if $x \in W_{C}\left(A_{1}, \ldots, A_{p}\right)$, so is $-x$.

Theorem 7 is best possible in the sense that there are examples of $W_{C}\left(A_{1}, \ldots, A_{p}\right)$ which fail to be convex when $p>3$ and $n \geq 1$. When $n=1$, Lemma 6 is also valid for $W_{C}\left(A_{1}, \ldots, A_{p}\right)$, except that the matrix $A$ is $p \times 3$ and is formed from $A_{1}, \ldots, A_{p}$, i.e., $W_{C}\left(A_{1}, \ldots, A_{p}\right)$ is a 2 -ellipsoid in $\mathbb{R}^{p}$. So it is not always convex. When $n \geq 2$, it suffices to consider the case $p=4$. The following matrices are $k \times k$ skew symmetric matrices, where $k \geq 4$, even or odd.

\section{Example.}

$$
\begin{aligned}
C=\left(\begin{array}{cc}
0 & 1 \\
-1 & 0
\end{array}\right) \oplus 0, & \\
A_{1}=\left(\begin{array}{cc}
0 & 1 \\
-1 & 0
\end{array}\right) \oplus\left(\begin{array}{cc}
0 & -1 \\
1 & 0
\end{array}\right) \oplus 0, & A_{2}=\left(\begin{array}{cc}
0 & 1 \\
-1 & 0
\end{array}\right) \oplus\left(\begin{array}{cc}
0 & 1 \\
-1 & 0
\end{array}\right) \oplus 0, \\
A_{3}=\left(\begin{array}{cccc}
0 & 0 & 1 & 0 \\
0 & 0 & 1 & 0 \\
-1 & -1 & 0 & 0 \\
0 & 0 & 0 & 0
\end{array}\right) \oplus 0, & A_{4}=\left(\begin{array}{cccc}
0 & 0 & 0 & 1 \\
0 & 0 & 0 & 1 \\
0 & 0 & 0 & 0 \\
-1 & -1 & 0 & 0
\end{array}\right) \oplus 0 .
\end{aligned}
$$

The points $(-2,-2,0,0)$ and $(2,-2,0,0)$ are obviously in $W_{C}\left(A_{1}, A_{2}, A_{3}, A_{4}\right)$. However, $W_{C}\left(A_{1}, A_{2}, A_{3}, A_{4}\right)$ does not contain the midpoint $(0,-2,0,0)$. Indeed, otherwise there would exist $O \in S O(k)$ such that

$$
C^{\prime}=\left(O^{T} C O\right)[1,2,3,4 \mid 1,2,3,4]=\left(\begin{array}{cccc}
0 & \frac{1}{2} & \alpha & \beta \\
-\frac{1}{2} & 0 & -\alpha & -\beta \\
-\alpha & \alpha & 0 & \frac{1}{2} \\
-\beta & \beta & -\frac{1}{2} & 0
\end{array}\right)
$$

Since the rank of $C$ is 2 , the rank of the submatrix $C^{\prime}$ is not greater than 2 . Now the first two rows of $C^{\prime}$ are obviously linearly independent. The third row has to be a linear combination of the first two rows, i.e.,

$$
\left(-\alpha, \alpha, 0, \frac{1}{2}\right)=\kappa_{1}\left(0, \frac{1}{2}, \alpha, \beta\right)+\kappa_{2}\left(-\frac{1}{2}, 0,-\alpha,-\beta\right) .
$$

So $\kappa_{1}=\kappa_{2}=2 \alpha$ from the first two coordinates of both sides of the equality. But then the fourth coordinate will yield $\frac{1}{2}=0$. This is absurd. 


\section{Some Remarks AND QUESTIONS}

Question 1. If $G=S O(2 n)$, is there a convexity result when $p=3$ and $n \geq k$ for some $k$ ? If not, it would be interesting to have nonconvex examples when $n \geq 2$. Obviously, if $n=1, W_{C}\left(A_{1}, A_{2}, A_{3}\right)$ is a singleton set and hence is convex. If there is such convexity theorem for $S O(2 n)$ when $p=3$, then according to the example in the previous section ( $k$ can be even), it will be best possible in the same sense.

If $x, y \in \mathbb{R}^{n}$, we denote by $y \ll x$ the relation defined by the inequalities

$$
\begin{aligned}
\sum_{i=1}^{k}\left|y_{i}\right| & \leq \sum_{i=1}^{k}\left|x_{i}\right|, \quad k=1, \ldots, n, \\
\sum_{i=1}^{n-1}\left|y_{i}\right|-\left|y_{n}\right| & \leq \sum_{i=1}^{n-1}\left|x_{i}\right|-\left|x_{n}\right|,
\end{aligned}
$$

and in addition, if the total number of negative terms of the sequences $x$ and $y$ is odd,

$$
\sum_{i=1}^{n}\left|y_{i}\right| \leq \sum_{i=1}^{n-1}\left|x_{i}\right|-\left|x_{n}\right|
$$

after rearranging the sequences $x$ and $y$ in decreasing order with respect to the absolute values.

The geometry behind the partial ordering $\ll$ is like that behind $\prec$ and $\prec_{w}$, namely, $y \ll x$ if and only if $y$ is in the convex hull of the vectors $\left( \pm c_{\theta(1)}, \ldots, \pm c_{\theta(n)}\right)$, where $\theta \in \Sigma_{n}$ and the number of negative signs is even [12].

Lemma 5 provides a characterization of the partial relation $\prec_{w}$ which is useful to the proof of our main result. See [13] for the statements equivalent to the convexity of $W_{C}\left(A_{1}, A_{2}, A_{3}\right)$ when $G=S O(2 n)$. The partial order $\ll$ is involved.

Question 2. Is there a characterization of $\ll$ similar to those of $\prec\left(x, y \in \mathbb{R}^{n},[8]\right)$ and $\prec_{w}\left(x, y \in \mathbb{R}_{+}^{n}\right.$, Lemma 5) ?

\section{ACKNOWLEDGEMENT}

Thanks are given to the referee for helpful suggestions.

\section{REFERENCES}

[1] M. F. Atiyah and R. Bott, The Yang-Mills equations over Riemann surfaces, Philos. Trans. Roy. Soc. London Ser. A 308 (1983), 523-615. MR 80k:14006

[2] Y. H. Au-Yeung and Y. T. Poon, A remark on the convexity and positive definitness concerning Hermitian matrices, Southeast Asian Bull. Math. 3 (1979), 85-92. MR 81c: 15026

[3] Y. H. Au-Yeung and N. K. Tsing, Some theorems on the generalized numerical ranges, Linear and Multilinear Algebra, 15 (1984), 3-11. MR 85c:15039

[4] Y. K. Au-Yeung and N. K. Tsing, An extension of the Hausdorff-Toeplitz theorem on the numerical range, Proc. Amer. Math. Soc. 89, (1983), 215-218. MR 85f:15021

[5] K. M. Chong, An induction theorem for rearrangements, Canad. J. Math. 28 (1976), 154-160. MR 52:14200

[6] K. E. Gustafson and D. K. M. Rao, Numerical Range: the field of values of linear operators and matrices, Springer, New York, 1997. CMP 97:03

[7] A. W. Marshall and I. Olkin, Inequalities: Theory of majorization and its applications, New York: Academic Press, 1979. MR 81b:00002

[8] R. F. Muirhead, Some methods applicable to identities and inequalities of symmetric algebraic functions of $n$ letters, Proc. Edinburgh Math. Soc. 21 (1903), 144-157.

[9] L. Mirsky, On a convex set of matrices, Arch. Math. 10 (1959), 88-92. MR 21:5643 
[10] Y. T. Poon, Another proof of a result of Westwick, Linear and Multilinear Algebra, 9 (1980), 35-37. MR 81h:15015

[11] Y. T. Poon, Generalized numerical ranges, joint positive definiteness and multiple eigenvalues, Proc. Amer. Math. Soc. 125 (1997), 1625-1634. MR 97g:15030

[12] T.Y. Tam, Kostant's convexity theorem and the compact classical groups, Linear and Multilinear Algebra 43 (1997), 87-113.

[13] T.Y. Tam, Generalized numerical ranges, numerical radii, and Lie groups, manuscript, 1996.

[14] T.Y. Tam, Plotting the generalized numerical range associated with the compact connected Lie groups, manuscript, 1996.

[15] R. Westwick, A theorem on numerical range, Linear and Multilinear Algebra 2 (1975), 311315. MR 51:11132

Department of Mathematics, Auburn University, Auburn, Alabama 36849-5310

E-mail address: tamtiny@mail.auburn.edu 